\title{
Extent of venous resection during pancreatectomy-finding the balance of technical possibility and feasibility
}

\author{
Atsushi Oba ${ }^{1}$, Tomotaka Kato ${ }^{1}$, Yosuke Inoue ${ }^{1}$, Y. H. Andrew Wu ${ }^{2}$, Yoshihiro Ono ${ }^{1}$, Takafumi Sato ${ }^{1}$, \\ Hiromichi Ito ${ }^{1}$, Akio Saiura ${ }^{3}$, Yu Takahashi ${ }^{1}$ \\ ${ }^{1}$ Division of Hepatobiliary and Pancreatic Surgery, Cancer Institute Hospital, Japanese Foundation for Cancer Research, Tokyo, Japan; ${ }^{2}$ Department \\ of Surgery, Johns Hopkins University School of Medicine, Baltimore, MD, USA; ${ }^{3}$ Department of Hepatobiliary-Pancreatic Surgery, Juntendo \\ University School of Medicine, Tokyo, Japan \\ Contributions: (I) Conception and design: A Oba, T Kato, Y Inoue, A Saiura, Y Takahashi; (II) Administrative support: None; (III) Provision of study \\ material or patients: None; (IV) Collection and assembly of data: None; (V) Data analysis and interpretation: A Oba, T Kato, Y Inoue, YHA Wu, Y \\ Takahashi; (VI) Manuscript writing: All authors; (VII) Final approval of manuscript: All authors. \\ Correspondence to: Atsushi Oba, MD, PhD. Division of Hepatobiliary and Pancreatic Surgery, Cancer Institute Hospital, Japanese Foundation for \\ Cancer Research, 3-8-31 Ariake, Koto-ku, Tokyo 135-8550, Japan. Email: atsushi.oba@jfcr.or.jp.
}

\begin{abstract}
The improvement of effective multidrug agents has allowed more patients to undergo resection for pancreatic cancer (PC). In the conversion cases of initially unresectable PC after induction chemotherapy, pancreatic surgeons often encounter challenging vein resections cases such as those of long-segment portal vein (PV)/superior mesenteric vein (SMV) encasement or occlusion of the distal (caudal) SMV. Given the lack of consensus for the optimal approach for major vein resections and reconstructions in these situations, this review summarizes the literature on this topic and provides the best currently available approaches for challenging vein reconstruction cases. For long-segment PV/SMV encasement, tips for direct end-to-end anastomosis without grafts and the splenic vein $(\mathrm{SpV})$ reconstruction to prevent left-side portal hypertension will be introduced. For distal SMV encasement, several bypass techniques to deal with collateralizations will be introduced. Even though some high-volume PC centers are obtaining favorable outcomes for challenging vein resection cases, existing evidence on this topic is limited. It is essential to organize the well-designed international multicenter studies for the small population of challenging vein resection cases. With the emergence of effective chemotherapies, the number of PC patients who can undergo curative resection is increasing. Achieving more successful vessel resection and reconstruction in the treatment of PC is a common goal that pancreatic surgeons should focus on together.
\end{abstract}

Keywords: Pancreatic cancer (PC); vein resection; locally advanced pancreatic cancer; neoadjuvant treatment; left-side portal hypertension

Submitted Mar 10, 2021. Accepted for publication Jul 23, 2021.

doi: 10.21037/jgo-21-129

View this article at: https://dx.doi.org/10.21037/jgo-21-129

\section{Introduction}

Curative resection has continuously provided the best longterm prognosis for pancreatic cancer (PC) (1). As pancreatic tumors easily invade major mesenteric vessels, artery or vein resection and reconstruction are often required to achieve curative resection for advanced PC cases (2). Since Dr. Fortner and colleagues first reported the concept and results of "regional pancreatectomy", extensive pancreatectomies combined with major vascular resection and reconstruction have been gradually introduced and improved around the world $(2,3)$. The introduction of effective multi-agent drugs, such as FOLFIRINOX or gemcitabine/nab-paclitaxel serves as a major turning point in modern treatment for PC as pancreatectomies with vein resections have become more actively adopted at high-volume centers, accomplishing low rates of mortality $(0.7-5.2 \%)(4,5)$. According to the current 
A

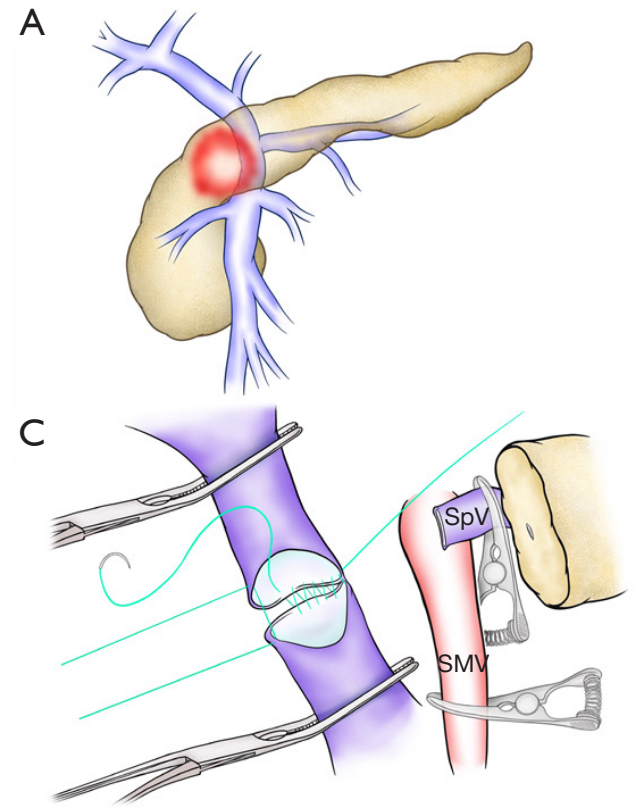

B
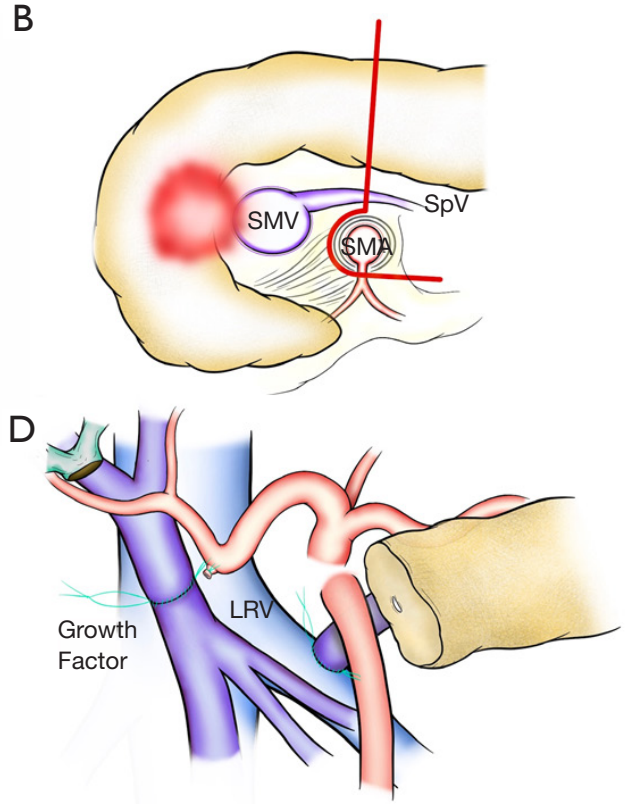

Figure 1 Long-segment PV/SMV encasement. (A) A tumor with proximal SMV involvement. (B) The Concept of "regional pancreaticoduodenectomy". The retropancreatic segment of the porto-mesenterico-splenic confluence and surrounding soft tissue are resected en bloc. The pancreas is divided along the line above the superior mesenteric artery. (C) The parachute technique. The right and left walls are sutured by 5-0 Proline without tying. From left to right, the posterior wall is very loosely running sutured to minimize tension on the vein wall. After securing the posterior wall with adequate stitches, the anastomosis of the posterior wall would be accomplished by pulling each thread towards the other side along with the orifice. (D) An end-to-side anastomosis of the $\mathrm{SpV}$ to the LRV. PV, portal vein; SMV, superior mesenteric vein; SpV, splenic vein; LRV, left renal vein; SMA, superior mesenteric artery.

National Comprehensive Cancer Network guidelines, the cases of technically challenging vein reconstruction are categorized as borderline resectable or locally advanced PCs (6). Recently, Kinny-Köster et al. from the Johns Hopkins group categorized two challenging situations: long-segment portal vein (PV)/superior mesenteric vein (SMV) encasement, occlusion of the distal (caudal) SMV even with involvement of the jejunal and ileal branches (7). In these situations, cavernous transformation and venous collateralization often introduces surgical challenges due to the high likelihood of encountering increased blood loss and congestion of the intestinal veins and small bowel (Figures 1,2). The optimal approaches for these vein resection challenges have been rarely discussed and the published outcomes are limited. This paper reviews the best currently available approaches for challenging vein reconstruction cases and summarizes the findings from existing literature.

\section{Technically possible situations}

\section{Long-segment PV/SMV encasement}

The length of the major veins involved by cancer is critical for vein reconstruction and resection. The approach and current consensus for a $20-\mathrm{mm}$ vein reconstruction and $50 \mathrm{~mm}$ vein reconstruction could be totally different. Current literature suggests unanimous agreement with performing end-to-end anastomosis for $20 \mathrm{~mm} \mathrm{PV/}$ SMV resection (8-13). Fujii et al. analyzed 197 patients with vein resection and revealed that direct end-to-end anastomosis was safe and offered patients improved rates of curative resection (8). However, cases that require $\geq 31 \mathrm{~mm}$ vein resection should consider the adoption of a graft to achieve tension-free anastomosis (8). Similarly, Dua et al. queried 90 patients who underwent vein resection and concluded that direct end-to-end anastomosis has superior results in achieving patency than the alternatives 
A

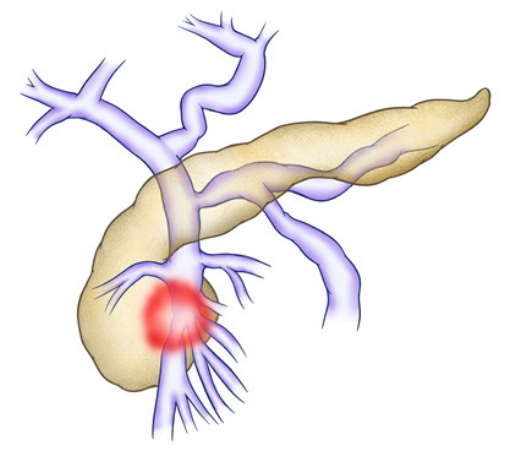

C

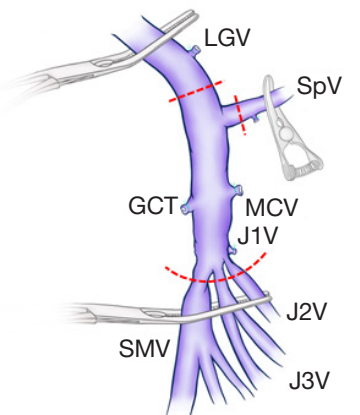

B
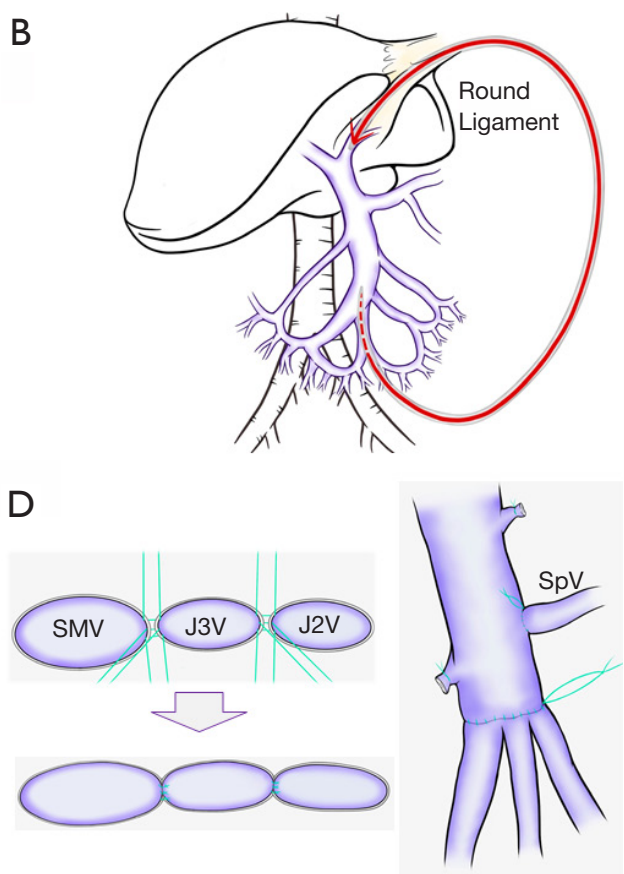

Figure 2 Distal SMV involvement. (A) A tumor involving distal SMV including the second jejunal vein or further branches. (B) The anthron bypass tube. One side of the catheter is inserted into the distal jejunal or ileal branches, while the other side is inserted into the round ligament that connects the umbilical portal vein. (C) The proximal and distal SMV and the SpV are clumped and divided, and the tumor is removed. Resection of the $\mathrm{SpV}$ can be performed to release the tension at the SMV anastomosis. (D) A single SMV lumen is created by unifying two or three of SMV side of branches by interrupted or continuous sutures. After performing the SMV anastomosis, the $\mathrm{SpV}$ is reconstructed to the PV or the LRV. SMV, superior mesenteric vein; LGV, left gastric vein; GCT, gastrocolic trunk; MCV, middle colic vein; J1V, the first jejunal vein; J2V, the second jejunal vein; J3V, the third jejunal vein; SpV, splenic vein; PV, portal vein; LRV, left renal vein.

after PV/SMV resection and should be the preferred technique for short segment $(<30 \mathrm{~mm})$ reconstructions (9). Terasaki et al. compared 97 patients who underwent direct end-to-end anastomosis and 25 patients who underwent an interposition graft placement using the right external iliac vein (10). The same study emphasized that using the right external iliac vein as an interposition graft is safe and effective since direct end-to-end anastomosis and graft interposition in patients with PC showed no differences in short-term outcomes. Recently, Labori et al. reported a systematic review of the use of four graft types (autologous veins, synthetic grafts, cadaveric allografts, and parietal peritoneum/falciform ligament) for PV/SMV resection (14). Even though the rest three types seem to have a lower risk of early graft thrombosis ( $<30$ days) $(2.5-6.7 \%)$ than that of synthetic grafts $(7.5 \%)$, thrombosis occurs with all types of grafts.
Considering this concern, some surgeons might prefer to adopt the direct end-to-end anastomosis approach for $5 \mathrm{~cm}$ or more PV/SMV resections (5,11-13). Zhang et al. reported that direct end-to-end anastomosis for 5 to $7 \mathrm{~cm}$ of long-segment PV/SMV resection by performing tension-reducing liver mobilization and the CattellBraasch maneuver at the anastomotic site was possible without encountering complications (11). Wang et al. also emphasized that direct end-to-end anastomosis can be performed safely even when the length of PV/SMV resection is $4 \mathrm{~cm}$ or more (12). Del Chiaro et al. queried 45 patients who underwent pancreaticoduodenectomy (PD) or total pancreatectomy with vein resection, combining a Cattell-Braasch maneuver. The median length of PV/ SMV resection segment was $46 \mathrm{~mm}$ (range, 30-70 mm) and all the cases in the study underwent a direct end-to-end anastomosis without graft interposition (13). 
In our recent cohort, except one patient who had an interposition graft, 90 patients (99\%) achieved a direct endto-end anastomosis of the PV/SMV stumps without any mortality and thrombus (Figure 1B,C,D). Median length of resected vein was $40 \mathrm{~mm}$ (range, $20-80 \mathrm{~mm}$ ). Antiplatelet or anticoagulation therapy was rarely used (5). We would like to present two approaches that aim for higher success in direct end-to-end anastomosing of long segmental veins. First, active division of the splenic vein $(\mathrm{SpV})$ could achieve the mobilization of the PV and the SMV with a tensionfree anastomosis without causing a stricture or thrombus. Second, if necessary, we actively adopt parachute technique in order to prevent the vein wall from tearing due to tension in one place (15). In brief, the right and left walls are sutured by 5-0 Proline (Ethicon Inc., Cornelia, GA) without tying. From left to right, the posterior wall is very loosely running sutured to keep the tension-free to the vein wall (Figure 1C). After securing the posterior wall with adequate stitches, the anastomosis of the posterior wall would be accomplished by pulling each thread towards the other side along with the orifice. The anterior wall anastomosis is performed by running the suture from left to right, as in a usual direct end-to-end anastomosis. Finally, two threads from the posterior and anterior wall are tied. The growth factor equivalent to the diameter of the reconstructed vein is preserved for later vessel expansion. As previously reported in literature, liver mobilization and the CattellBraasch maneuver would also work.

\section{SpV reconstruction to prevent left-side portal hypertension}

The risk of left-side portal hypertension after surgery is a major drawback of dividing the $\mathrm{SpV}$ (16). We previously reported that among 43 patients who underwent $\mathrm{PD}$ with $\mathrm{SpV}$ division, 26 patients (62.8\%) developed gastrointestinal varices due to left-side portal hypertension with followup CT findings (16). Several papers demonstrated the $\mathrm{SpV}$ can be divided without reconstruction by ligating the splenic artery or decompensating of the left gastric vein or other veins (the middle colic vein, the inferior mesenteric vein etc.) (17-21). Although, we further reported 5 out of 44 patients $(11 \%)$ with postoperative gastrointestinal varices experienced life-threatening rupture (22), these data were validated by a multi-center study later. In this cohort, among 227 patients who underwent $\mathrm{PD}$ with $\mathrm{SpV}$ division, varices development was detected in 84 patients $(37.0 \%)$, moreover, variceal bleeding occurred in 9 patients (4.0\%) (23).
Although the necessity of the reconstruction of the $\mathrm{SpV}$ is very debatable and surgeons' opinions regarding the $4 \%$ of the chance of variceal bleeding may vary (17-23), we highly recommend $\mathrm{SpV}$ reconstruction $(24,25)$. Our current first choice is the end-to-side anastomosis of the $\mathrm{SpV}$ to the left renal vein (or the PV), due to the higher patency rate and feasible technique compared to other endto-end anastomoses with or without interposition graft (24) (Figure 1D).

Recently, our group reported a method of measuring the $\mathrm{SpV}$ pressure during surgery using 27 -gauge needles. In this study, high SpV pressure after clamping ( $\geq 20 \mathrm{mmHg}$ ) and a large $\mathrm{SpV}$ pressure difference $(\geq 10 \mathrm{mmHg})$ before and after clamping are indication criteria for $\mathrm{SpV}$ reconstruction to prevent left-side portal hypertension (26).

\section{Distal SMV involvement}

The cases of distal (caudal) SMV involvement are more challenging than those of the porto-mesentericosplenic confluence involvement $(7,25)$. First, there are fewer distal SMV involvement cases than the portomesenterico-splenic confluence involvement cases. Second, the involvement or occlusion of distal SMV with the highly possible involvement of the jejunal and ileal branches (which are thinner than the root of SMV) may cause the subsequent development of collaterals that make tumor removal difficult. Collateralizations increase the risk of the bleeding and congestion of mesenteric venous flow and small bowel during resection. Finally, the reconstruction of distal SMV, which is smaller and thinner than proximal SMV, is technically demanding. The John Hopkins team introduced several bypass reconstructions such as mesoportal bypass, interposition graft, and mesocaval shunt during resection (7). Similarly, Oehme et al. reported the usefulness of portosystemic shunts (a splenorenal shunt or a temporary mesocaval shunt) during extended pancreatectomy. This approach also prevents left-side portal hypertension and enables complete tumor resection even for the cases with developed cavernous transformation (27). Our approach to these situations involves performing a simpler temporary bypass intraoperatively (25). To further limit intraoperative bleeding and ensure safe anastomosis in distal SMV occlusion cases, our team adopt the use of antithrombogenic catheter (Anthron Bypass Tube, Toray Industries, Inc., Tokyo, Japan) that was originally introduced by Nakao et al. (28). One side of the catheter 
is inserted into the distal jejunal or ileal branches, while the other side is inserted into the round ligament that connects the umbilical PV (or otherwise the femoral vein via the greater saphenous vein, or inferior vena cava via the gonadal vein). Based on our experience, this procedure confers the advantage of avoiding harvesting autologous grafts, such as the internal jugular vein, the left renal vein or the saphenous vein. Due to its cost and lack of practicality in some institutions, homologous vein may not be the most viable option to ensure a bypass or reconstruction without occlusion (29). The removal of the Anthron catheter can be easily performed by ligating the round ligament when resection and reconstruction are complete. On the other hand, with venous obstruction and significant venous collateral developments, vein resection without reconstruction was initially introduced as a novel approach in locally advanced PCs (due to non-constructible vein involvement) (30). Kulkarni et al. performed vein resection without reconstruction for six patients and emphasized the need to assess venous collateralization pre- and intraoperatively (30). Since the evidence of vein resection without reconstruction is not well established, we prefer to perform reconstruction whenever it is feasible. Figure $2 C, 2 D$ is the representative images that depict the reconstruction for the distal SMV occlusion case. For tumors that invade the distal SMV extending to the peripheral side of the $\mathrm{J} 2 \mathrm{~V}$, we prefer resecting the $\mathrm{SpV}$ to release the tension of SMV anastomosis and creating a single SMV lumen by unifying two or three of SMV side of branches by interrupted or continuous sutures. After performing the SMV anastomosis, we prefer reconstructing the $\mathrm{SpV}$ to the left renal vein (or the PV).

\section{Biological aspect}

A recent meta-analysis showed that vein resection is associated with higher morbidities, mortalities, and has no benefit for survival compared to standard PD (31). The median overall survival in the study was reported to be 14.3 months for patients who underwent pancreatectomy with PV/SMV resection and 19.5 months for those without vein resection $(\mathrm{P}=0.063)$. Although, as the authors mentioned, since there were theoretically more advanced diseases in the vein resection group, this comparison might undermine the benefit of vein resection in achieving improved long-term survival outcomes for advanced PC patients (31). In fact, the degree of venous invasion reflects the prognosis. Nakao et al. classified the radiographic types of vein invasion of PC patients into A (normal), $\mathrm{B}$ (unilateral narrowing), $\mathrm{C}$ (bilateral narrowing), or D (complete obstruction with collateral veins), and revealed the prognosis is significantly different between type A vs type $\mathrm{B}(\mathrm{P}=0.011)$ and type $\mathrm{B}$ vs type $\mathrm{C} / \mathrm{D}(\mathrm{P}=0.0022)$, respectively (32). Similarly, Murakami et al. queried multicenter database and showed overall survival of PC with vein contact of $\leq 180$ degree group was significantly better than that of PC with vein contact of $\geq 180$ degree group (median survival time after resection: 22.0 vs. 17.4 months, respectively) (33).

Our group have actively adopted vein resection, [62.2\% (268 out of 431 cases) of overall pancreatic head tumors cases] not only for removing the tumors invading vein, but also for attempting to achieve en bloc resection and negative surgical margin, i.e. tumors surrounding soft tissue. This aggressive strategy could be justified by the favorable short-term outcomes of low mortality, low rate of thrombosis, and better long-term prognosis (the median overall survival: 25.7 months) (5). Similarly, Terasaki and colleagues performed 135 concomitant vein resections out of 225 overall PDs for PC (60.0\%) and reported excellent short- and long-term outcomes with only $0.8 \%$ of patient experiencing stenosis within 30 days postoperatively and $0.8 \%$ of mortality after vein resection (10). Given there are no randomized controlled trials of comparing vein resection versus non-surgery approach after neoadjuvant treatment, we need to accumulate favorable short-term results and potentially better long-term outcomes after vein resection. From this point of view, it should be emphasized here that aggressive vein resection should ideally only be performed at a high-volume center.

\section{Long-segment PV/SMV encasement}

As far as we know, no study compares the long-term outcomes by the length of vein segment directly. However, good long-term outcomes were witnessed in studies that included groups with longer median vein lengths $(4.0-4.6 \mathrm{~cm})(5,10,13)$. Del Chiaro et al. reported 45 patients who underwent $\mathrm{PD}$ with vein resection, and the mean length of the resected PV/SMV segment was $4.6 \mathrm{~cm}$ (range, $3.0-7.0 \mathrm{~cm}$ ). The $1-$ and 3 -year survival rate was $70.7 \%$ and $28 \%$, respectively, with the median survival time of 27 months (13). Among 122 PC patients with vein resection of Terasaki's cohort, there 
was no significant difference in the survival between the interposition graft group (the 2- and 5-year survival rates: $32.0 \%$ and $10.0 \%$ ) and the end-to-end anastomosis group (24.5\% and $13.7 \%, \mathrm{P}=0.963$ ), even though length of $\mathrm{PV}$ resection was longer in the interposition graft group than in the end-to-end anastomosis group [4.5 (2.0-7.0) vs. $3.0(0.5-5.5) \mathrm{cm}, \mathrm{P}<0.001](10)$. Our group recently introduced new radical technique of "regional PD" (5). The concept of this procedure is leaving the retropancreatic segment of the porto-mesenterico-splenic confluence and surrounding soft tissue untouched (en bloc), and dividing the pancreas along the line above the superior mesenteric artery (SMA) (Figure 1B). In this paper, although the length of resected PV/SMV segment was significantly greater for regional PD $[4.0 \mathrm{~cm}(2.0-8.0 \mathrm{~cm})]$ than that of standard $\mathrm{PD}$ with vein resection $[3.0 \mathrm{~cm}(1.0-6.0 \mathrm{~cm}), \mathrm{P}<0.001]$, the overall survival after regional PD (32 months) was better than in the standard PD group (21 months, $\mathrm{P}=0.004)$. These three papers indicated that the longer segment vein resection was not related to poorer long-term survival and imply that pancreatic surgeons can perform aggressive radical pancreatectomy with long vein resection.

\section{Distal SMV involvement}

There are fewer reports of long-term results for distal SMV resection. Honda et al. reported 10 patients with resection of the second jejunal vein or further branches of the SMV (J2VR) (34). The outcomes were compared with the rest 104 patients with PV/SMV resection above J2V (standard PVR). R0 rate (the J2VR vs standard PVR: $90 \%$ vs. $88 \%$ ), thrombosis (10\% vs. $1 \%)$, and 2 -year survival rates $(67 \%$ and $45 \%$ ) were not significantly different between two groups. They demonstrated that PD with J2VR can be safely achieved with a favorable prognosis. To date, the result in this paper is the only evidence that show the longterm survival outcome of the distal SMV reconstruction cases compared to the normal vein reconstruction cases, despite a small sample size of 10 cases. Even though the details of tumor location for all patients were not presented, Gage et al. reported the overall survival of 5 locally advanced PC cases without vein reconstruction (35). These patients underwent PD with distal SMV resection after neoadjuvant treatment, with meticulous attention to hemostasis and preservation of collateral venous flow. Their survival time after surgery and from diagnosis were 5-24 and 17-32 months, respectively.

\section{Conclusions: where we are?}

Even though some high-volume PC centers around the world are obtaining excellent short- and long-term outcomes for challenging vein resection PC cases, existing evidence on this topic is limited. Even if tumor location on the pancreas were same, the degree of vein obstruction may vary depending on the aggressiveness of the tumor or the degree of contact to the SMV, and the collateralization may vary depending on the mesentery anatomy (the branching of LGV, IMV, and the superior right colic vein arcade) (16). It is important to note that it is difficult to develop an optimal strategy for small population of patients who present challenging vein invasion. There is no doubt that a good short-term prognosis is the starting point for discussing long-term prognosis. For that purpose, a well-organized patient selection is necessary. This includes surgical treatment strategies even before neoadjuvant chemotherapy, understanding precise anatomy based on multidetector computed tomography, and evaluating patient's conditional status.

It is essential to organize the well-designed international multicenter studies for the small population of challenging vein resection cases. With the emergence of effective chemotherapies, the number of PC patients who can undergo curative resection is increasing. Achieving more successful vessel resection and reconstruction in the treatment of PC is a common goal that pancreatic surgeons should focus on together.

\section{Acknowledgments}

Funding: None.

\section{Footnote}

Provenance and Peer Review: This article was commissioned by the Guest Editor (Elena Rangelova) for the series "Surgery for Locally Advanced Pancreatic Cancer" published in Fournal of Gastrointestinal Oncology. The article has undergone external peer review.

Conflicts of Interest: All authors have completed the ICMJE uniform disclosure form (available at https://dx.doi. org/10.21037/jgo-21-129). The series "Surgery for Locally Advanced Pancreatic Cancer" was commissioned by the editorial office without any funding or sponsorship. The 
authors have no other conflicts of interest to declare.

Ethical Statement: The authors are accountable for all aspects of the work in ensuring that questions related to the accuracy or integrity of any part of the work are appropriately investigated and resolved.

Open Access Statement: This is an Open Access article distributed in accordance with the Creative Commons Attribution-NonCommercial-NoDerivs 4.0 International License (CC BY-NC-ND 4.0), which permits the noncommercial replication and distribution of the article with the strict proviso that no changes or edits are made and the original work is properly cited (including links to both the formal publication through the relevant DOI and the license). See: https://creativecommons.org/licenses/by-nc-nd/4.0/.

\section{References}

1. Oba A, Ho F, Bao QR, et al. Neoadjuvant Treatment in Pancreatic Cancer. Front Oncol 2020;10:245.

2. Oba A, Bao QR, Barnett CC, et al. Vascular Resections for Pancreatic Ductal Adenocarcinoma: Vascular Resections for PDAC. Scand J Surg 2020;109:18-28.

3. Fortner JG. Regional resection of cancer of the pancreas: a new surgical approach. Surgery 1973;73:307-20.

4. Peng C, Zhou D, Meng L, et al. The value of combined vein resection in pancreaticoduodenectomy for pancreatic head carcinoma: a meta-analysis. BMC Surg 2019;19:84.

5. Oba A, Ito H, Ono Y, et al. Regional pancreatoduodenectomy versus standard pancreatoduodenectomy with portal vein resection for pancreatic ductal adenocarcinoma with portal vein invasion. BJS Open 2020;4:438-48.

6. NCCN Clinical Practice Guidelines in Oncology: pancreatic adenocarcinoma, version 1.2020. 2020.

7. Kinny-Köster B, van Oosten F, Habib JR, et al. Mesoportal bypass, interposition graft, and mesocaval shunt: Surgical strategies to overcome superior mesenteric vein involvement in pancreatic cancer. Surgery 2020;168:1048-55.

8. Fujii T, Nakao A, Yamada $\mathrm{S}$, et al. Vein resections $>3 \mathrm{~cm}$ during pancreatectomy are associated with poor 1-year patency rates. Surgery 2015;157:708-15.

9. Dua MM, Tran TB, Klausner J, et al. Pancreatectomy with vein reconstruction: technique matters. HPB (Oxford) 2015;17:824-31.

10. Terasaki F, Fukami Y, Maeda A, et al. Comparison of end-to-end anastomosis and interposition graft during pancreatoduodenectomy with portal vein reconstruction for pancreatic ductal adenocarcinoma. Langenbecks Arch Surg 2019;404:191-201.

11. Zhang J, Qian HG, Leng JH, et al. Long mesentericoportal vein resection and end-to-end anastomosis without graft in pancreaticoduodenectomy. J Gastrointest Surg 2009;13:1524-8.

12. Wang F, Arianayagam R, Gill A, et al. Grafts for mesenterico-portal vein resections can be avoided during pancreatoduodenectomy. J Am Coll Surg 2012;215:569-79.

13. Del Chiaro M, Segersvärd R, Rangelova E, et al. CattellBraasch Maneuver Combined with Artery-First Approach for Superior Mesenteric-Portal Vein Resection During Pancreatectomy. J Gastrointest Surg 2015;19:2264-8.

14. Labori KJ, Kleive D, Khan A, et al. Graft type for superior mesenteric and portal vein reconstruction in pancreatic surgery - A systematic review. HPB (Oxford) 2021;23:483-94.

15. Nanashima A, Abo T, Kunizaki M, et al. Portal vein anastomosis with parachute method in hepatectomy and pancreatectomy. Hepatogastroenterology 2012;59:1000-2.

16. Ono Y, Matsueda K, Koga R, et al. Sinistral portal hypertension after pancreaticoduodenectomy with splenic vein ligation. Br J Surg 2015;102:219-28.

17. Tanaka H, Nakao A, Oshima K, et al. Splenic vein reconstruction is unnecessary in pancreatoduodenectomy combined with resection of the superior mesenteric veinportal vein confluence according to short-term outcomes. HPB (Oxford) 2017;19:785-92.

18. Strasberg SM, Bhalla S, Sanchez LA, et al. Pattern of venous collateral development after splenic vein occlusion in an extended Whipple procedure: comparison with collateral vein pattern in cases of sinistral portal hypertension. J Gastrointest Surg 2011;15:2070-9.

19. Rosado ID, Bhalla S, Sanchez LA, et al. Pattern of Venous Collateral Development after Splenic Vein Occlusion in an Extended Whipple Procedure (Whipple at the Splenic Artery) and Long-Term Results. J Gastrointest Surg 2017;21:516-26.

20. Gyoten K, Mizuno S, Nagata M, et al. Significance of Simultaneous Splenic Artery Resection in Left-Sided Portal Hypertension After Pancreaticoduodenectomy with Combined Portal Vein Resection. World J Surg 2017;41:2111-20.

21. Yamada D, Takahashi H, Hama N, et al. The clinical impact of splenic artery ligation on the occurrence of 
digestive varices after pancreaticoduodenectomy with combined portal vein resection: a retrospective study in two institutes. Langenbecks Arch Surg 2021;406:1469-79.

22. Tanaka M, Ito H, Ono $Y$, et al. Impact of portal vein resection with splenic vein reconstruction after pancreatoduodenectomy on sinistral portal hypertension: Who needs reconstruction? Surgery 2019;165:291-7.

23. Mizuno S, Kato H, Yamaue H, et al. Left-sided Portal Hypertension After Pancreaticoduodenectomy With Resection of the Portal Vein/Superior Mesenteric Vein Confluence in Patients With Pancreatic Cancer: A Project Study by the Japanese Society of Hepato-BiliaryPancreatic Surgery. Ann Surg 2021;274:e36-44.

24. Ono Y, Tanaka M, Matsueda K, et al. Techniques for splenic vein reconstruction after pancreaticoduodenectomy with portal vein resection for pancreatic cancer. HPB (Oxford) 2019;21:1288-94.

25. Ito H, Takahashi Y, Saiura A. Commentary: Complex venous resection and reconstruction for locally advanced pancreatic cancer: Our approach. Surgery 2020;168:1056-7.

26. Ono Y, Takahashi $Y$, Tanaka $M$, et al. Sinistral Portal Hypertension Prediction During Pancreatoduodenectomy With Splenic Vein Resection. J Surg Res 2021;259:509-15.

27. Oehme F, Distler M, Müssle B, et al. Results of portosystemic shunts during extended pancreatic resections. Langenbecks Arch Surg 2019;404:959-66.

28. Nakao A, Nonami T, Harada A, et al. Portal vein resection with a new antithrombogenic catheter. Surgery 1990;108:913-8.

Cite this article as: $\mathrm{Oba} \mathrm{A}$, Kato $\mathrm{T}$, Inoue $\mathrm{Y}, \mathrm{Wu}$ YHA, Ono $\mathrm{Y}$, Sato T, Ito H, Saiura A, Takahashi Y. Extent of venous resection during pancreatectomy-finding the balance of technical possibility and feasibility. J Gastrointest Oncol 2021;12(5):24952502. doi: $10.21037 /$ jgo-21-129
29. Yamamoto M, Akamatsu N, Aoki T, et al. Safety and efficacy of cryopreserved homologous veins for venous reconstruction in pancreatoduodenectomy. Surgery 2017;161:385-93.

30. Kulkarni RV, Patil V, Bhandare MS, et al. Vein resection without reconstruction (VROR) in pancreatoduodenectomy: expanding the surgical spectrum for locally advanced pancreatic tumours. Langenbecks Arch Surg 2020;405:929-37.

31. Giovinazzo F, Turri G, Katz MH, et al. Meta-analysis of benefits of portal-superior mesenteric vein resection in pancreatic resection for ductal adenocarcinoma. Br J Surg 2016;103:179-91.

32. Nakao A, Kanzaki A, Fujii T, et al. Correlation between radiographic classification and pathological grade of portal vein wall invasion in pancreatic head cancer. Ann Surg 2012;255:103-8.

33. Murakami Y, Satoi S, Sho M, et al. National Comprehensive Cancer Network Resectability Status for Pancreatic Carcinoma Predicts Overall Survival. World J Surg 2015;39:2306-14.

34. Honda M, Nagakawa Y, Akashi M, et al. Clinical impact of pancreaticoduodenectomy for pancreatic cancer with resection of the secondary or later branches of the superior mesenteric vein. J Hepatobiliary Pancreat Sci 2020;27:731-8.

35. Gage MM, Reames BN, Ejaz A, et al. Pancreaticoduodenectomy with en bloc vein resection for locally advanced pancreatic cancer: a case series without venous reconstruction. Chin Clin Oncol 2018;7:7. 